\title{
Nonlinear Modeling of Dynamic Cerebral Autoregulation Using Recurrent Neural Networks*
}

\author{
Max Chacón ${ }^{1}$, Cristopher Blanco ${ }^{1}$, Ronney Panerai ${ }^{2}$, and David Evans ${ }^{2}$ \\ ${ }^{1}$ Informatic Engineering Department, University of Santiago de Chile, \\ Av. Ecuador 3659, PO Box 10233, Santiago, Chile \\ mchacon@diinf.usach.cl, christopher.blanco@usach.cl \\ ${ }^{2}$ Medical Physics Group, Department of Cardiovascular Sciences, University of Leicester, \\ Leicester Royal Infirmary, Leicester LE1 5WW, UK \\ \{rp9, dhe\}@le.ac.uk
}

\begin{abstract}
The function of the Cerebral Blood Flow Autoregulation (CBFA) system is to maintain a relatively constant flow of blood to the brain, in spite of changes in arterial blood pressure. A model that characterizes this system is of great use in understanding cerebral hemodynamics and would provide a pattern for evaluating different cerebrovascular diseases and complications. This work posits a non-linear model of the CBFA system through the evaluation of various types of neural networks that have been used in the field of systems identification. Four different architectures, combined with four learning methods were evaluated. The results were compared with the linear model that has often been used as a standard reference. The results show that the best results are obtained with the FeedForward Time Delay neural network, using the Levenberg-Marquardt learning algorithm, with an improvement of $24 \%$ over the linear model $(\mathrm{p}<0.05)$.
\end{abstract}

\section{Introduction}

The determination of a model for the Cerebral Blood Flow Autoregulation (CBFA) system, is an important physiological modeling problem, not only because of the importance of maintaining Cerebral Blood Flow (CBF) within narrow limits, but also because this flow self regulation system is present in a number of other organs [1].

The CBFA system has been the object of intense study recently, due to the development of transcranial Doppler as a tool for measuring CBF Velocity (CBFV), which can be assumed to be the equivalent to CBF [1,2]. A key element in the modeling of CBFA is the determination of the dynamic relationship that exists between Arterial Blood Pressure (ABP) and CBFV. The cerebral autoregulation literature includes a wide variety of studies that seek to characterize this relationship. In general these studies try to induce abrupt changes in patient $\mathrm{ABP}$ and then measure the effect of these changes on CBFV [1,3-4]. A non-invasive alternative, of great clinical importance in the modeling of CBFA is to analyze the relationship between $\mathrm{ABP}$ and $\mathrm{CBFV}$ by observing natural oscillations or spontaneous fluctuations in $\mathrm{ABP}$

${ }^{*}$ This study has been supported by FONDECYT (Chile) project $\mathrm{N}^{\circ} 1050082$. 
and its effect on CBFV. The validity of this type of analysis was demonstrated previosuly [1,4-6]. This technique can be applied both to healthy and sick patients including premature neonates. Thus, the greatest possibilities of generating clinical tools lie in the use of models based on spontaneous fluctuations.

In order to characterize the ABP-CBFV relationship by measuring spontaneous fluctuations of $\mathrm{ABP}$, a series of signal processing techniques have been used. Among these are transfer functions models, frequency analysis and impulse and step responses [4, 6-9]. Studies in the time domain have also been carried out using moving average filters such as the Wiener-Laguerre $[4,6]$ and differential equations as proposed by Aaslid-Tiecks [6-10]. These models assume a linear relation between $\mathrm{ABP}$ and CBFV, which severely limits the results obtained, due to the existence of a number of non-linearities in the system [6] which are not considered in linear models. Up to now, there have been few attempts to model the system with non-linear methods. To our knowledge, there are only three studies that used neural networks [11-13]. Mitsis et al [11] used spontaneous fluctuations, but proposed a model based on a particular type of neural network. They have studied only five subjects and did not compare their findings with other models, such as the commonly used AaslidTiecks linear model [6-10]. Thus, there is a dearth in the development of non-linear models of CBFA, especially with signals that facilitate the construction of diagnostic methods.

The present work proposes the modeling of the ABP-CBFV relationship through the use of non-linear system identification techniques, which hitherto have not been applied to autoregulation modeling. The different network architectures analyzed in the present work were: FeedForward Time Delay, Neural Net Output Error, Elman Net and Time Lagged FeedForward. These architectures were combined with the following learning methods: Backpropagation with momentum, Delta Bar Delta, Levenberg-Marquardt and One Step Secant [14-17]. Moreover, the results obtained with neural networks were compared with results obtained with the Aaslid-Tiecks model $[1,3,6,10]$, the most often used linear model in the field of cerebral hemodynamics for evaluating autoregulation.

\section{Methods}

\subsection{Data Collection and Pre-processing}

The study included 16 volunteer subjects with no history of cardio-vascular or neurologic disease. The average \pm standard deviation (SD) age was $30 \pm 7$ years, with a range of 23 to 47 years. Measurements were carried out at Leicester Royal Infirmary in a room with a temperature of approximately $23^{\circ} \mathrm{C}$. The study was approved by the Leicestershire ethics committee and written consent was obtained from each subject.

CBFV was monitored in the medial cerebral artery using a Scimed QVL-120 Transcranial Doppler with a $2 \mathrm{MHz}$ transducer. APB was measured with a non-invasive Finapres 2300 Ohmeda monitor.

Pressure and CBFV data were collected and stored on digital audio tape with a Sony PC108M 8-track recorder for posterior processing. 
The data on the tape were transferred to a microcomputer in real time. FFT was used to extract the maximum velocity with a time window of $5 \mathrm{~ms}$. The ABP signal was sampled at 200 samples/sec. Both signals were filtered with a Butterworth $8^{\text {th }}$ order low pass filter with a cut off frequency of $20 \mathrm{~Hz}$. The beginning of each cardiac cycle was detected from the end-diastolic value of the ABP wave, and mean values for ABP and $\mathrm{CBFV}$ were calculated for each cardiac cycle.

The data obtained from the samples were conditioned for further analysis with the models. The measures of ABP and CBFV for each patient consisted of a file with approximately 1500 samples taken every 0.2 seconds. Given that the Nyquist frequency for these signals is approximately $1 \mathrm{sec}$. it is possible to determine the mean of three samples, thus obtaining a sampling period of 0.6 seconds. In this way, the number of samples was reduced to 500 per patient.

\subsection{Neural Network Models}

Neural networks require short term memories in order to represent dynamic systems. Networks that include such memories are known as recurrent neural networks. These networks can be divided into two large groups, static neuronal networks with external recurrence, and networks with internal memory.

Networks with external recurrence are basically multilayer perceptrons which retard some of their inputs, and re-input their output with some delay. In this particular project we used FeedForward Time Delay and Neural Net Output Error networks which are representative of this type of model (also called NARX networks [18]).

Networks with internal memory attempt to extend the duration of short term memory provided by networks with external recurrence. In order to achieve this extension, so-called context memories are introduced. In this work two types of networks with internal memory were used: Elman Net and Time Lagged FeedForward.

The general structure for FeedForward Time Delay (FFTD) networks used for the CBFA model, is presented in Figure. 1.

An important characteristic of these networks, is the manner in which training is carried out. In contrast to the training of a static network, these networks follow a strict sequence for the order in which signal samples are presented.

The structure of a Neural Net Output Error (NNOE) [19-20] is essentially a FFTD network to which an error term is added that is represented by a white noise signal, the purpose of which is to represent a non-explained portion of the variance of the output variable, (in this case the CBFV). The inclusion of a signal that complements the chosen variables for this model allows a better fit between said model and the data being considered.

The Elman Net (Elman) [14-17] has a classic recurrent neural network topology, and has all the elements that are typical of a multilayer perceptron, which turn it into a universal function approximator. Moreover, it has context units which represent a recurrent link with the hidden layer. This property allows the network to address an important limitation of static neural networks with external recurrences which is the definition of a fixed time window that does not allow the gathering of information about samples that occurred at time prior to $D=\max \{N, M\}$. 


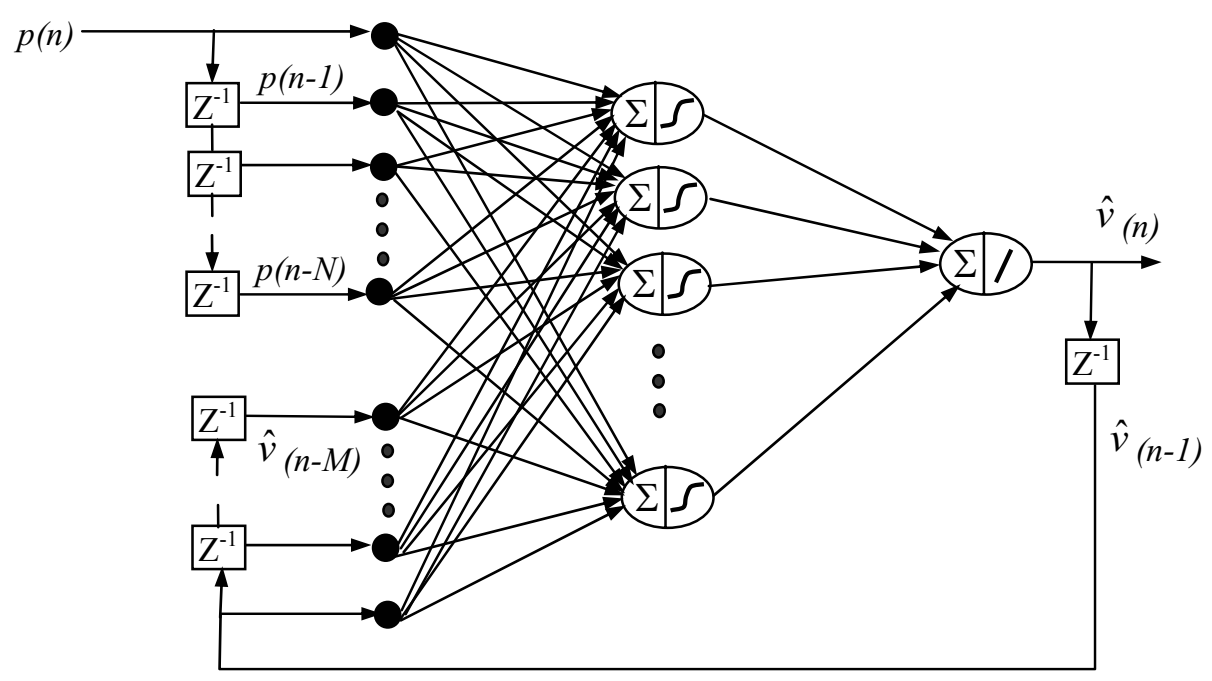

Fig. 1. Architecture of the FeedForward Time Delay (FFTD) neural network

Context memories can not only store information from the last $D$ samples, but can also incorporate information from all the samples that the network has seen This is due to the fact that their output is generated as a function of the weighting of all the samples that have been seen during the network's training. The basic structure for context memories is shown in Figure 2.

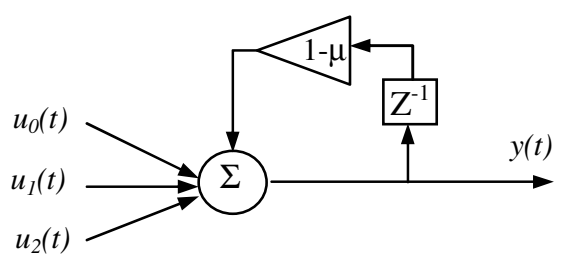

Fig. 2. Context memory for the Elman network

The principal shortcoming of these memories is that they do not deliver the exact value of the last $D$ samples, these values are only approximated. In exchange, however, they are able to store approximate information about samples that have been seen outside the window of $D$ samples. The memory parameter $\mu$ must be adjusted, making a compromise between the detail with which the memory recalls certain samples, and the number of samples in the past that it is able to recall.

An important property of the Elman network is that, because it has a fixed recurrent link, it can be easily trained using a variation of the Backpropagation algorithm [14-15]. This model does not require that the delay number be explicitly indicated either for the input or for the output; determining the number of neurons in the hidden layer is sufficient. 
Time Lagged FeedForward (TLFF) networks use a topology that is similar to that of FFTD, but they include context units known as Gamma and Laguerre memories [14], considered to be more powerful than those used in Elman networks.

Figure $3 \mathrm{a}$ and $3 \mathrm{~b}$ show Gamma and Laguerre memories. These memories are essentially a cascade of low-pass filters in the case of the Gamma memory, and band pass filters for the Laguerre memory (except for the first), all of which have a common parameter $\mu$. When only one memory is used, this is the same as used in the Elman network. When $\mu$ is 1 , the memory becomes a line of simple delays such as used in FFTD networks. Both memories have the same representational capacity. The difference lies in the response to impulse of the Laguerre memory which becomes more oscillatory than the Gamma memory. Nonetheless, the Laguerre memory has the advantage that it stabilizes in less time than the Gamma memory.

The different learning methods or algorithms evaluated, correspond to variations and improvements on the backpropagation algorithm. A momentum term is added to this classic algorithm to improve convergence. The second improvement consists of adopting variable linear learning rates for individual weights. In this case the Delta Bar Delta algorithm linearly increases the rate of learning when the sign of the slope does not vary, and reduces the rate exponentially when it does.

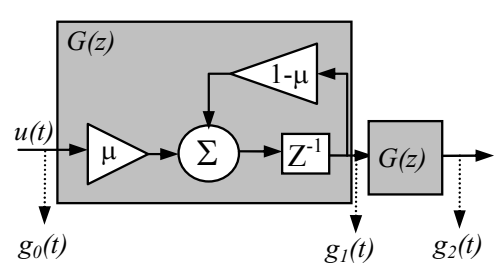

(a)

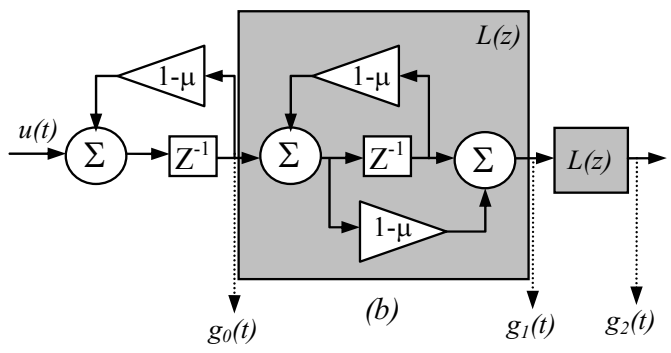

Fig. 3. a) Gamma Memory, b) Laguerre Memory, for the TLFF network

The other two algorithms correspond to quasi-Newton methods that avoid having to calculate the Hessian matrix. The Levenberg-Marquardt algorithm uses a combination of Jacobian matrices, which can be calculated with the Standard Backpropagation algorithm. The One Step Secant method uses an approximation of the combined Hessian matrix by calculating the direction of search of the slope which uses the directions of the previous slopes.

\subsection{Selection of Parameters and Statistical Analysis}

The different topologies were evaluated using a hidden layer [21], in which the number of neurons used varied from 2 to 20. The number of input and output signal delays varied from zero to 20 delays for each architecture, with the exception of the Elman network. Furthermore, to estimate the order of the system, the method proposed by He and Asada [20] was used, and an empirical verification was carried out to coincide with the combination that yields the best performance. The methods evaluated for stopping training were Early Stopping and Regularization [14-15]. 
The signals for each patient were divided into two groups of 2.5 minutes each. Thus, a crossed validation strategy was developed $[14,16,22]$ in which a model was generated for the patient with the first part of the signal, evaluated by the second part, and vice-versa. In total, 32 models were generated for each topology used.

In order to measure the performance of the models, the correlation coefficient $(r)$ was used as well as the normalized mean-square error (NMSE). The latter is defined as the sum of the squares of the error between the model's output $(\hat{v})$ and the real output value $(v)$ divided by the square of the real value of the output $[11,13]$.

In order to compare different model results in terms of error and correlation, Wilcoxon's non-parametric sign test was used, and two results were considered significantly different when $p<0.05$.

Further to comparing the results obtained with the different alternatives of nonlinear networks, the data were evaluated with Aaslid-Tiecks [10] linear model, which estimated CBFV using a second degree linear differential equation made up of two state variables. The relevance of this lies in the consideration of physiological elements in the estimation of autoregulation of the subjects. Additionally, this model provides an index of dynamic autoregulation which allows the individual performance of CBFA to be assessed on a scale of 1 to 10 .

\section{Results}

The different models were tested on 16 subjects, and the architecture that generated the best average result for all subjects was selected. For each architecture, (except the Elman network, which only determines the number of neurons in the hidden layer), the fundamental parameters that were adjusted were: the number of delays applied to the ABP (PD) signal, the number of delays applied to the CBFV (VD) signal, and the

Table 1. Mean results for the different models, best architecture, best learning method, NMSE, and correlation coefficient $(r)$ for training and testing

\begin{tabular}{ccc|cc|ccc}
\hline Model & \multicolumn{2}{c}{ Architecture Learning } & \multicolumn{2}{c}{ Training } & \multicolumn{2}{c}{ Testing } \\
& PD-VD-HL & Method & NMSE \pm SD & r \pm SD & \multicolumn{1}{c}{ NMSE \pm SD } & r \pm SD \\
\hline Linear & $2-2$ & - & $62.3 \% \pm 30.2 \%$ & $0.55 \pm 0.15$ & $66.5 \% \pm 33.1 \%$ & $0.51 \pm 0.20$ \\
FFTD & $8-2-6$ & LM & $51.5 \% \pm 30.5 \%$ & $0.65 \pm 0.14$ & $57.3 \% \pm 25.3 \%$ & $0.63 \pm 0.13$ \\
NNOE & $6-2-10$ & LM & $36.8 \% \pm 12.9 \%$ & $0.73 \pm 0.09$ & $78.8 \% \pm 64.4 \%$ & $0.58 \pm 0.09$ \\
Elman & 20 & Elman & $51.5 \% \pm 16.5 \%$ & $0.60 \pm 0.13$ & $63.2 \% \pm 23.0 \%$ & $0.52 \pm 0.16$ \\
TLFF & $8-6-12$ & DBD & $48.1 \% \pm 8.4 \%$ & $0.56 \pm 0.18$ & $48.3 \% \pm 8.9 \%$ & $0.53 \pm 0.20$ \\
\hline
\end{tabular}

number of neurons in the hidden layer (HL). Table 1 shows the best average results for each model, including the Aaslid-Tiecks linear model [6-10]. Upon analyzing the results presented in Table 1, it is evident that the best model depends on the evaluation parameter being considered. We believe that due to the large variability present in the spontaneous fluctuation signals, the best parameter for evaluation is the correlation coefficient obtained with the test set, and that NMSE should be used only as a means of comparison with other works. 
Figure 4 shows a typical CBFV prediction for a patient using the FFTD model (with the LM algorithm), who shows the highest mean correlation value in testing.

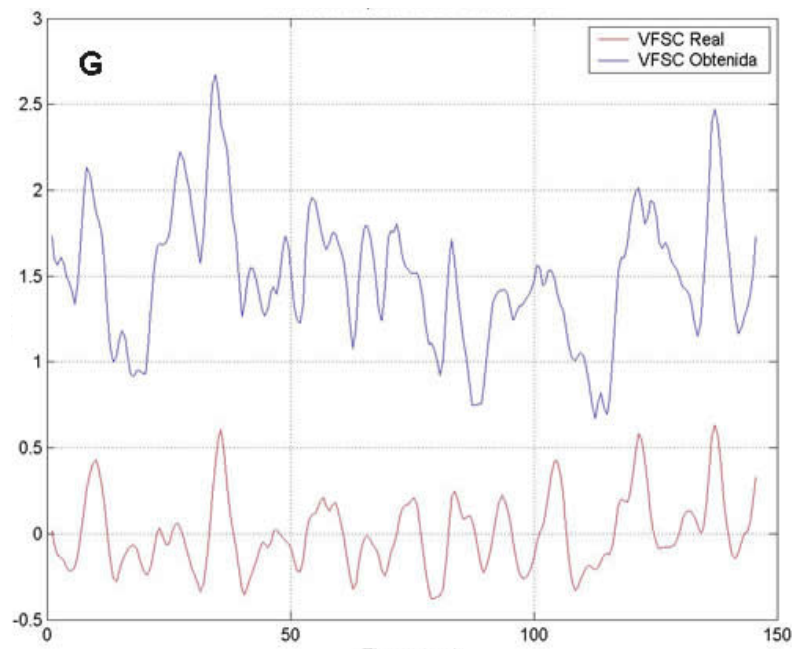

Fig. 4. Prediction for a CBFV segment with the FFTD model, and the LM learning algorithm, real signal $v$ (above) and prediction $\hat{v}$ (below)

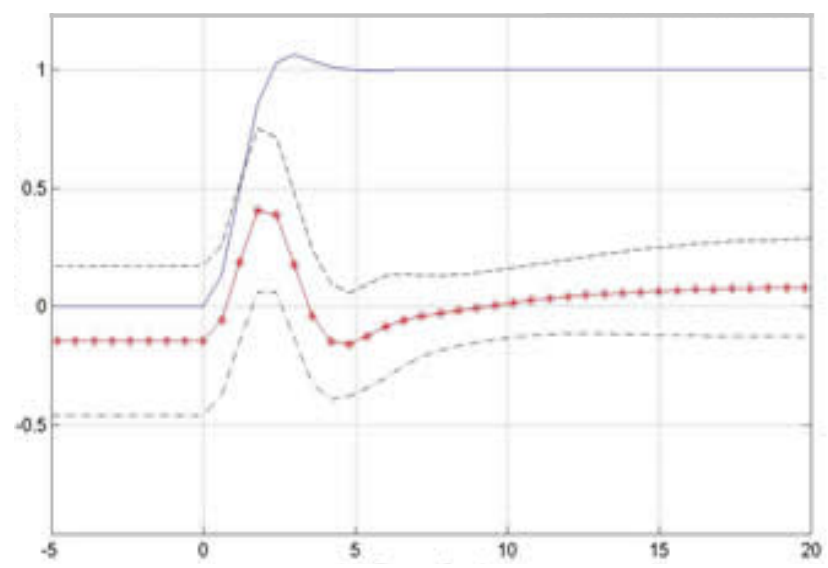

Fig. 5. Mean response of $\mathrm{CBFV}$ to the $\mathrm{ABP}$ step for 16 subjects. ABP step (solid line), mean CBFV response (asterisks), $\pm \mathrm{CBFV}$ standard deviation (dotted line).

An important characteristic for evaluating a CBFA model is determining whether the model is able to capture the physiological dynamics of the CBFA system, or if the model has only managed a numerical fit for the data. One way of determining whether this is the case, or not, is to input an ABP step signal that simulates a thigh cuff technique, to an already trained model, and examine the CBFV response signal. Figure 5 shows the ABP step signal and the mean CBFV response for the various models for 16 subjects. 


\section{Discussion and Conclusions}

Upon examining the correlations coefficients for the test set in Table 1, it is evident that the two models that noticeably surpass the correlation achieved by the linear model are the FFTD model (0.12 higher) and the NNOE model (0.07 higher).

Sign test results for the FFTD network with LM were significantly superior to the linear model. The next model that shows large differences with the linear model is the NNOE model, but its hypothesis test showed that the difference was not significant $(p=0.010)$. The better performance of the non-linear models can also be seen when comparing them against the two linear models used by Panerai [6,8-9]. This shows that CBFA is a non-linear phenomenon.

In order to compare these results with non-linear models, we can refer to the work done by Mitsis et al [11], which is the only work that uses the same type of signal. Their study achieved an NMSE of $27.6 \% \pm 9.5 \%$, for its non-linear solution, which is inferior to our results. However, there are two important differences to consider which can narrow the difference between these results. Mitsis et al. analysed only 5 subjects, and they calculated the NMSE over the entire signal, whereas our errors correspond exclusively to the test set.

The mean response of CBFV to the models shown in Figure 5 represent an adequate physiological response to the ABP step signal, indicating that the non-linear model not only achieves a numerical fit, but also represents the normal physiological function of the CBFA system in healthy subjects.

The high correlation values of the NNOE model over the training set should be highlighted. The principal difference between this model and the others, is that it considers a white noise signal for modeling, which represents those elements that can affect CBFV and which cannot be completely represented by the ABP signal. Future work should consider the possibility of including other variables such as the partial pressure of carbon dioxide $\left(\mathrm{pCO}_{2}\right)$ and critical closing pressure [23].

The importance of the present work resides in that it proves, generally speaking, the superiority of neural network models in the modeling of CBFA. However, it leaves unaddressed a great deal of challenges, such as the evaluation with other signal types (thigh cuff technique and valsalva maneuver), the generation of multivariate models, and the development of classification systems to allow clinical assessment of CBFA in patients.

\section{References}

1. Panerai R. Assessment of cerebral pressure autoregulation in humans - a review of measurement methods. Physiological Measurement 19 (1998) 305-38.

2. Newell D, Aaslid R, Lam A, Mayberg T, Winn R. Comparison of flow and velocity during autoregulation testing in humans. Stroke 25 (1994) 793-7.

3. Panerai R, Evans D, Mahony P, Deverson S, Hayes P. Assessment of thigh cuff technique for measurement of dynamic cerebral autoregulation. Stroke 31 (2000)476-80.

4. Panerai RB, Dawson SL, Eames PJ and Potter JF. Cerebral blood flow velocity response to induced and spontaneous sudden changes in arterial blood pressure. Am J Physiol 280 (2001) H2162-H2174. 
5. Panerai R, Kelsall A, Rennie J, and Evans D. Cerebral autoregulation dynamics in premature newborns. Stroke 26 (1995) 74-80.

6. Panerai R, Dawson S, Potter J. Linear and nonlinear analysis of human dynamic cerebral autoregulation. Am J Physiol 227 (1999) H1089-H1099.

7. Panerai R, White R, Markus H, Evans D. Grading of cerebral dynamic autoregulation from spontaneous fluctuations in arterial blood pressure. Stroke 29 (1998) 2341-6.

8. Panerai R, Rennie J, Kelsall A, Evans D. Frequency-domain analysis of cerebral autoregulation from spontaneous fluctuations in arterial blood pressure. Med. Biol. Eng. Comput 36 (1998) 315-22.

9. Simpson D, Panerai R, Evans D, Naylor R. A Parametric Approach to Measuring Cerebral Blood Flow Autoregulation from Spontaneous Variations in Blood Pressure. Annals of Biomedical Engineering 29 (2001)18-25.

10. Tiecks F, Lam A, Aaslid R, Newell D. Comparison of static and dynamic cerebral autoregulation measurements. Stroke 26 (1995) 1014-9.

11. Mitsis G, Zhang G, Levine BD, Marmarelis VZ. Modeling of Nonlinear Physiological Systems with fast and Slow Dynamics. II. Application to cerebral Autoregulation. Ann. Biomedical Engineering 30 (2002) 555-65.

12. Panerai R, Chacón M, Pereira R and Evans D. Neural Network Modeling of Dynamic Cerebral Autoregulation: Assessment and Comparison with Established Methods. Med Eng \& Phys 26 (2004) 43-52.

13. Mitsis G, ZPoulin M Robbins A Marmarelis VZ. Nonlinear Modeling of the Dynamic Effects of Arterial Pressure and CO2 Variations on Cerebral Blood Flow in Healthy Humans. IEEE Trans. Bio. Eng 51 (2004) 1932-43.

14. Principe J, Euliano N, Lefebvre C. Neural and Adaptive Systems: Fundamentals Through Simulations. New York: John Wiley \& Sons (2000).

15. Hilera J, Martínez V. Redes Neuronales Artificiales. Fundamentos, modelos y aplicaciones. Madrid: RA-MA (1995).

16. Bishop C. Neural Networks for Pattern Recognition. Oxford: Oxford University Press (1995).

17. Elman J. Finding Structure in Time. Cog. Sci 4 (1990) 141-66.

18. Lin T, Horne B, Tiňo P, Giles C. Learning Long-Term Dependencies in NARX Recurrent Neural Networks, IEEE Trans. on Neu. Net. 7 (1996) 1329-38.

19. Ljung L. System Identification - Theory for the User. New Yersey: Prentice Hall (1987).

20. He X, Asada H. A New Method for Identifying Orders of Input-Output Models for Nonlinear Dynamics Systems. Proc. of the American Control Conf, S F California (1993).

21. Hornick K, Stinchcombe M, White H. Multilayer feedforward networks are universal approximators. Neural Networks 2 (1997) 359-66.

22. Mitchell T. Machine Learning. New York: WCB/McGraw-Hill (1997).

23. Panerai R.B. The critical closing pressure of the cerebral circulation. Med. Eng. \& Phy. 25 (2003) 621-32. 